\title{
Diacronie
}

Studi di Storia Contemporanea

$N^{\circ} 24,4 \mid 2015$

Le dittature militari: fisionomia ed eredità politica

\section{Diana Napoli, Michel de Certeau. Lo storico "smarrito"}

\section{Enrico Castelli di Gattinara}

\section{(2) OpenEdition}

\section{Journals}

\section{Edizione digitale}

URL: http://journals.openedition.org/diacronie/3593

DOI: $10.4000 /$ diacronie.3593

ISSN: 2038-0925

\section{Editore}

Association culturelle Diacronie

Notizia bibliografica digitale

Enrico Castelli di Gattinara, "Diana Napoli, Michel de Certeau. Lo storico "smarrito" », Diacronie [Online],

$N^{\circ} 24,4$ | 2015, documento 20, Messo online il 29 décembre 2015, consultato il 24 septembre 2020.

URL : http://journals.openedition.org/diacronie/3593; DOI : https://doi.org/10.4000/diacronie.3593 


\section{Diacronie}

N. 24 | 4|2015 Le dittature militari: fisionomia ed eredità politica

20/

\section{RECENSIONE:}

\section{Diana NAPOLI, Michel de Certeau. Lo storico “smarrito", Brescia, Morcelliana, 2014, 248}

\section{pp.}

a cura di Enrico CASTELLI DI GATTINARA*

Nessuno può dire con precisione chi fosse intellettualmente Michel de Certeau: uno storico? Un religioso? Uno studioso della mistica? Un epistemologo della storia? Un antropologo? Un appassionato di psicanalisi? Era infatti un po' tutte queste cose insieme e forse l'appellativo di "studioso" - nel senso proprio e ormai antico del termine - sarebbe probabilmente il modo più adeguato per definirlo. Apprezzato a destra e a sinistra, dai cattolici e dagli atei, dai religiosi e dai laici, la sua figura di intellettuale si staglia con grande originalità nel panorama francese delle scienze umane della seconda metà del XX secolo. Gesuita per scelta e per convinzione, non fece mai pesare la propria religiosità come un a priori dogmatico a direzione dei propri studi e delle proprie curiosità intellettuali. Amava occuparsi infatti di ciò che sfugge alle definizioni (per questo è tanto difficile definire l'ambito specifico del suo lavoro intellettuale), delle differenze, dell'alterità irriducibile, di quanto resta senza parola, o assente, ma non per questo da considerare più irrilevante. Per farlo, si è voluto fare storico di fenomeni particolarmente difficili da storicizzare, come la mistica, che dell'assenza di parola e discorso fa la propria ragione e dell'abolizione del tempo storico la sua essenza. Di fronte a tale compito ha dovuto però costruire concetti operativi e approcci epistemologici di cui il sapere storico convenzionale era ancora sprovvisto, sostenendoli con una filosofia e un'ontologia capaci di renderne ragione. Ma in questo, era in buona compagnia.

Di questo sforzo a un tempo filosofico ed epistemologico tratta il recente, intenso libro di Diana Napoli, dedicato a Michel de Certeau (che avrebbe meritato una più attenta revisione redazionale, visti i numerosi e fastidiosi refusi). Non si tratta di una biografia - neppure di una biografia intellettuale, è bene dirlo subito - ma di un'analisi 
profonda e spesso difficile della complessità teorica del pensiero e dei lavori dello studioso francese nel contesto teorico, filosofico ed epistemologico (soprattutto francese, ma non solo) che va dagli anni Settanta alla fine degli anni Novanta del secolo scorso. Non si tratta neppure di un'analisi critica degli scritti e dei lavori dello storico francese, ma di una messa in gioco del suo approccio nell'ambito della storiografia e di un pensiero della storia di cui storici e filosofi negli ultimi decenni hanno riconosciuto lo stato critico. Si tratta di un lavoro importante, secondo il quale le opere di de Certeau non possono essere considerate semplicemente come dei meri studi storici o antropologici, perché le implicazioni filosofiche e ontologiche dei loro presupposti e delle loro istanze fondamentali, ma anche dei risultati ottenuti, sono imprescindibili per comprendere l'originalità di questo "storico smarrito" che può ancora indicarci una via per reagire alla perdita di identità (e di autorevolezza) cui è soggetta attualmente la storia.

È un libro di filosofia, quindi, che a partire dal pensiero di de Certeau spazia dalla storiografia all'estetica e dalla filosofia alla letteratura, coinvolgendo alcuni importanti autori francesi e tedeschi di riferimento (da P. Ricoeur a R. Koselleck, da S. Freud a G.W. Sebald) e s'interroga sullo statuto dello storico in un'epoca caratterizzata da quello che è comunemente definito, seguendo François Hartog, il "presentismo" (dove alla storia nella sua dinamica dialettica critica si è sostituita la memoria nella sua fissità memoriale intemporale, mentre tutta l'attenzione è orientata al presente, e non più al passato come esempio da seguire o al futuro come qualcosa da costruire secondo una linea di progresso) ${ }^{1}$.

I quattro capitoli in cui è diviso il libro, pur mossi da questo filo rosso conduttore che è il ruolo e lo statuto della ricerca storica di questi ultimi decenni, letto alla luce delle idee di de Certeau, sono molto diversi fra loro per contenuto. Il primo parla infatti esplicitamente dell'opera di Michel de Certeau, in particolare della sua opera di storico sui generis e del ruolo che vi ha giocato la rilettura di alcune delle tarde opere di Freud. Affronta con chiarezza la mai risolta questione della verità delle ricerche e delle scritture della storia. Nel secondo ci si concentra sul problema della Shoah e sulla difficoltà di una sua rappresentazione, anche come ricostruzione storica. Il terzo gira interamente intorno al problema del tempo, e in particolare della definizione del tempo storico. Il quarto infine analizza nei dettagli alcuni libri dello scrittore tedesco

\footnotetext{
1 HARTOG, François, Regimi di storicità. Presentismo ed esperienze del tempo, Palermo, Sellerio, 2007 [Ed. origi.: Régimes d'historicité. Présentisme et expériences du temps, Paris, Seuil, 2003].
} 
W.G.Sebald ${ }^{2}$, quasi a mettere alla prova quel modo assai particolare dell'operazione storiografica di cui de Certeau aveva mostrato lo stato di inquietudine e smarrimento essenziali e fondativi. Il passaggio stretto fra lo storico francese, il teorico tedesco e lo scrittore della distruzione mira, nei quattro capitoli, ad aprire «la possibilità di costruire una teoria dell'esperienza storica e indagare ulteriormente la relazione tra una teoria dell'esperienza storica e il significato attribuito alla scrittura della storia»3. La posta finale in gioco è infatti il significato che può avere ancora oggi la storiografia, nello smarrimento presentista in cui il reale ci si presenta come inesorabilmente frantumato, quasi senza memoria, ferito a morte e torturato ${ }^{4}$.

Lo spaesamento $^{5}$ cui conduce la particolare storia della mistica di Michel de Certeau è quell'inquietudine di cui aveva dato ragione Freud nella nozione di Unheimlich, che per Napoli è forse la chiave di volta dell'impalcatura storiografica decertiana. Il tema dello storico "smarrito" che a partire dal titolo attraversa tutto il libro' ${ }^{6}$ e che viene argomentato nel secondo capitolo, si basa sulla consapevolezza che il concetto di verità cui ci si era affidati per tanto tempo ormai non tiene più, e che il difficile - talvolta paradossale - compito dello storico di "ricostruire" o "rappresentare" il passato in un presente che non è mai uniforme né stabile è la scommessa che rende così sui generis lo statuto scientifico ed epistemologico della storia stessa. Ecco perché il primo capitolo spende i primi paragrafi sull' "antica questione dello statuto del "reale" rispetto alla scrittura della storia» e si chiede «in che relazione si trova la realtà con la scrittura storica che ne facciamo»7. Ecco anche perché il terzo capitolo introduce la riflessione accurata di Reinhart Koselleck, parzialmente ripresa da Paul Ricoeur e la cui tenuta viene illustrata attraverso alcuni "romanzi" di Sebald8.

Le questioni epistemologiche s’intrecciano per Napoli a quelle più propriamente filosofiche (ontologiche), per tradursi in una scrittura della storia che non è più meramente scientifica, né puramente letteraria, ma si tiene nel fragile equilibrio di una frattura mai del tutto consumata che traversa i diversi ambiti disciplinari. In questa frattura germoglia il discorso storico come discorso "altro", che si fa dell'altro, sull'altro e per l'altro. De Certeau aveva spiegato che la storia occupa un posto vuoto, quello

2 SEBALD, Winfried Georg, Storia naturale della distruzione, Milano, Adelphi, 2004 [Ed. originale: Luftkrieg und Literatur, Frankfurt am Main, Fischer, 2001]; ID., Austerlitz, Milano, Adelphi, 2002 [Ed. orig.: Austerlitz, Frankfurt am Main, Fischer, 2001].

3 NAPOLI, Diana, Michel de Certeau. Lo storico “smarrito", Brescia, Morcelliana, 2014, p. 13.

4 Ibidem, pp. 130-131, 182, 194, 222.

5 Ibidem, p. 121.

${ }^{6}$ Ibidem, pp. 12, 101, 109, 121, 179.

7 Ibidem, p. 34.

8 KOSELLECK, Reinhart, Futuro passato. Per una semantica dei tempi storici, Bologna, CLUEB, 2007; RICOEUR, Paul, La memoria, la storia, l'oblio, Milano, Cortina, 2003 [Ed. orig.: La mémoire, l'histoire, l'oubli, Paris, Seuil, 2000]. 
dell'assente, vale a dire il posto di quel passato che non può mai essere presente proprio perché già trascorso, e che la storia occupa paradossalmente riportandolo al presente con la sua scrittura, pur lasciandolo passato. Operazione storiografica che lo storico francese definiva anche come "menzognera", perché il passato ricondotto al presente dalla scrittura della storia resta assente, nascosto dall'operazione scritturale stessa. Di qui la situazione paradossale cui lo storico non può sfuggire. Di qui anche il suo smarrimento, che è però un vettore di forza e non una sconfitta (secondo la lezione freudiana). L'assenza «che permette al discorso storiografico di prodursi» si nutre di una realtà che è fatta sì di assenza, ma che non per questo è meno reale: solo che il suo statuto di verità deve essere discusso e compreso secondo una logica, o un'epistemologia che non corrisponde più al realismo ingenuo e ostensivo del "questo è questo", della concordanza fra la parola e la cosa, dell'esperienza totale e perfettamente costruita (o ri-costruita).

Richiamandosi a quell'esperienza inaudita e impercorribile sperimentalmente che è stata la Shoah, Napoli mostra come l'oggetto della storia - esemplarmente in questo caso - sia un'assenza che non può essere mai presa in una presenza, che è pressoché impossibile dire o ridire, e la cui scrittura non può farsi che per lacune, fratture e lacerazioni. Come Freud, nel suo libro su Mosè, aveva mostrato che «la separazione che "altera" si racconta unicamente attraverso una finzione, circonfusa di verità se si riconosce, abitata dalla menzogna quando (necessariamente) si nega come tale», così la storiografia «è la condizione che autorizza a costruire per l'altro un luogo altro, per il passato un luogo altro rispetto al presente»9. Ecco lo spaesamento, che nel caso della Shoah diventa emblematico. "Qui non c'è verità», come avvertiva Primo Levi riportando le parole di una SS del campo di sterminio: c'è solo una frattura fra testimonianza e documentazione, ognuna con le proprie verità non necessariamente coincidenti, da cui segue l'insufficienza di ogni rappresentazione ${ }^{10}$. Per gestire la problematizzazione di questo vero e proprio "oggetto del secolo", secondo la definizione di Gérard Wajcman ${ }^{11}$, l'autrice mobilita S. Friedländer, G. Agamben, P. Ricoeur, C. Lanzmann, R. Hilberg, Y.H. Yerushalmi e altri che argomentano la fragilità della rappresentazione storica, la quale si fa e si disfa in continuazione, sottraendosi in ultima istanza, ma facendo di questa sottrazione (in quanto assenza) la forza propulsiva del lavoro storiografico. Se la Shoah mostra tragicamente la non-coincidenza tra fatti e

9 Ibidem, p. 66. Cfr. FREUD, Sigmund, Mosè e il monoteismo, Roma, Newton \& Compton, 2010. ${ }^{10}$ NAPOLI, Diana, op cit., p. 101.

${ }^{11}$ WAJCMAN, Gérard, L'objet du siècle, Lagrasse, Verdier, 1998. 
verità, fra constatazione e comprensione - per usare le parole di Agamben ${ }^{12}$ cui l'autrice si ispira - ciò non deve portare al disincanto o al fallimento del sapere storico, ma alla sua inesauribile ricerca, consapevole di non potersi mai esaurire. E alla ridefinizione del termine concettuale di verità, si dovrebbe aggiungere. Perché è il discorso dello storico a "risarcire" l'intestimoniabile dell'annientamento avvenuto, facendo della propria "debolezza" epistemologica una forza che è al tempo stesso scientifica ed etica13. Ecco la lezione che ci lasciano gli studi di de Certeau sulla mistica, secondo Napoli. Ed ecco anche la lezione che ci è suggerita da Friedländer ${ }^{14}$, quel senso di smarrimento che si rivela come essenziale al lavoro storiografico: «Una volta al lavoro, lo storico deve sapere che le domande che solleva non trovano sempre risposta, accompagnandosi piuttosto di un sentimento di smarrimento [...] che si rivela indispensabile per non cedere alle lusinghe di una conclusione facile» ${ }^{15}$.

La lezione finale di Sebald, che conclude il libro erinforza secondo l'autrice le tesi di François Hartog, è allora l'irriducibilità attuale dello scarto fra spazio d'esperienza e orizzonte d'attesa, che ha aperto l'abisso del "presentismo" e rappresenta la catastrofe di ogni storicità (per una temporalità appunto detemporalizzata in un eterno presente): di fronte ad essa, lo storico deve divenire capace di attraversare l'opacità del reale per vincere la resistenza del passato ${ }^{16}$ senza lasciarsi illudere dall'evidenza di una rappresentazione definitiva ricostruita discorsivamente, di cui la storia sarebbe la sola dispensatrice. Deve vincere l'abbaglio del presente riconoscendo quella molteplicità temporale che frattura l'unità del tempo unico, articolando le differenze anche là dove esse sembrano non esserci più.

La conclusione apre infine a una consapevolezza fiduciosa sul compito scientifico, etico e politico che ancora spetta allo storico nel suo smarrimento. «La storiografia è obbligata a riconoscere che il documento ch'essa analizza [...] non è lo specchio del reale» ${ }^{17}$, ma l'immagine mai definitiva di un reale in frammenti, che lascia dietrodi sé sempre e inevitabilmente un "resto", uno scarto, vale a direun'alteritàche continuerà ad agire nascostamente sul presente. Per questo lo storico dovrà farsi nomade, viandante, accogliendo l'instabilità come una condizione inevitabile ma propulsiva del suo stesso lavoro. La sua verità sarà quindi una verità transeunte, passeggera, da montare di volta

${ }_{12}$ AGAMBEN, Giorgio, Quel che resta di Auschwitz, Torino, Bollati Boringhieri, 1998; ID., Che cos'è il contemporaneo, Roma, Nottetempo, 2008.

13 NAPOLI, Diana, op. cit., p. 139.

14F RIEDLÄNDER, Saul, La Germania nazista e gli ebrei. Gli anni dello sterminio 1939-1945, Milano, Garzanti, 2009 [Ed. orig.: Das Dritte Reich und die Juden. Die Jahreder Vernichtung 1939-1945, München, Beck, 2006].

15 NAPOLI, Diana, op. cit., p. 142.

${ }^{16}$ Ibidem, p. 221.

${ }_{17}$ Ibidem, p. 222. 
in volta di nuovo (come aveva fatto A. Warburg), essa stessa opaca. La fragile frattura in cui s’insinua il discorso storiografico diventa così un punto su cui far leva per scardinare quel continuum che Walter Benjamin aveva già preso di mira ormai molto tempo fa. Il denso libro di Napoli si chiude insomma su una nota di ottimismo, nel cui orizzonte però non si delinea alcuna strada maestra da seguire. 


\section{* L'autore}

Enrico Castelli Gattinara, filosofo, è stato per anni docente di Epistemologia della storia all'Università degli Studi di Roma la Sapienza e professore invitato presso l'Ecole des Hautes Etudes en Sciences Sociales a Parigi. Dirige la rivista di filosofia, arte e letteratura «Aperture. Punti di vista a tema» e ha pubblicato numerosi libri e articoli su temi all'incrocio fra epistemologia, scienze ed estetica. Si occupa di epistemologia delle scienze umane.

URL: < http://www.studistorici.com/progett/autori/\#CastelliDiGattinara >

\section{Per citare questo articolo:}

CASTELLI DI GATTINARA, Enrico, «Recensione: Diana NAPOLI, Michel de Certeau. Lo storico "smarrito", Brescia, Morcelliana, 2014, 248 pp.», Diacronie. Studi di Storia Contemporanea : Le dittature militari: fisionomia ed eredità politica, 29/12/2015,

URL:<http://www.studistorici.com/2015/12/29/castelli-digattinara_numero_24/ >

Diacronie Studi di Storia Contemporanea www.diacronie.it

Risorsa digitale indipendente a carattere storiografico. Uscita trimestrale.

redazione.diacronie@hotmail.it

Comitato di redazione: Jacopo Bassi - Luca Bufarale - Elisa Grandi - Antonio César Moreno Cantano - Deborah Paci - Fausto Pietrancosta - Alessandro Salvador - Matteo Tomasoni - Luca Zuccolo

Diritti: gli articoli di Diacronie. Studi di Storia Contemporanea sono pubblicati sotto licenza Creative Commons 3.0. Possono essere riprodotti e modificati a patto di indicare eventuali modifiche dei contenuti, di riconoscere la paternità dell'opera e di condividerla allo stesso modo. La citazione di estratti è comunque sempre autorizzata, nei limiti previsti dalla legge. 